\title{
Thermal Radiation Effects on MHD with Flow Heat and Mass Transfer in Casson Nanofluid over A Stretching Sheet
}

\author{
Yap Bing Kho ${ }^{l}$, Abid Hussanan ${ }^{1}$, Norhafizah Mohd Sarif ${ }^{1}$, Zulkhibri Ismail ${ }^{1}$ and Mohd Zuki Salleh ${ }^{{ }^{*}}$ \\ ${ }^{1}$ Applied \& Industrial Mathematics Research Group, Faculty of Industrial Science \& Technology, Universiti Malaysia Pahang, \\ 26300 UMP Kuantan, Pahang, Malaysia
}

\begin{abstract}
The boundary layer heat and mass transfer flow of Casson nanofluid over a stretching sheet with constant wall temperature (CWT) under the magnetic field and thermal radiation effects is investigated numerically. Using similarity transformations, the governing equations are reduced to a set of nonlinear ordinary differential equations (ODEs). These equations are solved numerically by Shooting method. The effects of Casson parameter, magnetic parameter, porosity parameter, radiation parameter, Prandtl number, Brownian parameter and thermophoresis parameter on velocity, temperature and concentration fields are shown graphically and discussed. The results show that increase in Casson parameter causes the wall temperature increase well in the nanofluid.
\end{abstract}

\section{Introduction}

Generally, the convectional fluids such as water, ethylene glycol and engine oil used in many industrial processes are poor heat conductors because these fluids have low thermal conductivities. To overcome this situation, Choi [1] was the first who introduced the idea of nanofluids. Nanofluids are a new class of fluids created by scattering nano/micro sized materials (nanoparticles, nanofibers, nanotubes, nanowires, nanorods and nanosheets) in conventional heat transfer fluids. Nanofluids are nanometer-sized particles with large surface area. Since the size of nanoparticles is in nanometer-sized, they have the ability to flow smoothly through the microchannel easily. After the first experimental work of Choi [1] on nanofluids, several researchers have used this concept in their convective heat transfer problems. Amongst them, Buongiorno [2] published a survey article on the convective transport in nanofluids. Ahmed and Pop [3] examined mixed convection flow of nanofluid which the model proposed by Tiwari and Das [4] with the condition that past over a vertical plate embedded in the porous medium. Yacob et al. [5] studied the boundary layer flow of a nanofluid over a stretching/shrinking sheet with convective boundary condition. Chamkha and Aly [6] used implicit finite difference method and analyzed the effect of heat generation or absorption on the steady free convection flow of a nanofluid over permeable vertical plate with suction or injection. Noghrehabadi et al. [7] studied partial slip effects on the boundary layer flow of nanofluid over a stretching sheet with constant wall temperature. Mahdy [8] studied mixed convection flow together with heat transfer in nanofluids due to stretching sheet. He found that the rate of heat transfer at the vertical stretching surface and thermal boundary layer of nanofluids increased as compared to pure water. Qasim et al. [9] studied heat and mass transfer phenomenon in nanofluids with convective boundary conditions whereas Loganathan and Vimala [10] obtained numerical solution of unsteady nanofluids flow lies over vertical flat plate with accretion or ablation at leading edge. Recently, Ganga et al. [11] studied the thermal radiation effects on hydromagnetic flow of a nanofluids and found the analytic solutions by using homotopy analysis method and numerical by the fourth order Runge-Kutta method.

MHD study on nanofluids with thermal effect over stretching sheet have play important rule in causes of high strength collision in plasma, resistivity and kinetic effect. The effects of magnetic field on heat transfer flow of a nanofluids lie over a vertical semi-infinite flat plate were investigated by Hamad [12]. Ellahi [13] had studied the analytical solution of non-Newtonian nanofluids inside a pipe with temperature dependent viscosity with the presence of MHD. Qasim et al. [14] used the conventional base fluid (water) with magnetite $\left(\mathrm{Fe}_{3} \mathrm{O}_{4}\right)$ nanoparticles and studied the ferrofluid flow of MHD and heat transfer analaysis over a stretched cylinder with given heat flux and slip condition. Hussanan et al. [15] considered unsteady MHD flow and heat transfer in some nanofluids over an accelerated vertical plate in a porous medium. They indicated that the effect of radiation parameter caused the wall temperature increased but temperature decreases significantly when Prandtl number is increased. Few other attempts made in this direction can be found in Krishna et al. [16] and Haq et al. [17].

All the above mentioned research however, focuses on Newtonian fluid (as water, ethylene glycol and engine oil) containing nanoparticles. Casson nanofluid is one of the fluids that do not obey Newton Laws and it is potentially useful in some applications, viz blood flow. Keeping this in view, Malik et al. [18] studied the Casson nanofluid with boundary layer over an

* Corresponding author: zuki@ump.edu.my 
exponentially stretching cylinder vertically and concluded that velocity profile drops as increase the Casson parameter.

As a continuation, our team propose here to study radiation and magnetic field effects on Casson nanofluid over a stretching sheet with constant wall temperature. The resulting governing equations are reduced to ordinary differential equations through similarity transformation and then solved numerically using the Shooting method.

\section{Mathematical Formulations}

The steady two dimensional boundary layer flow of nonNewtonian Casson nanofluid over a stretching plate embedded in a porous medium. The surface is stretching along $x$-axis with the velocity $U_{w}=a x$ at $y=0$, where $a>0$ is a constant. A constant magnetic field is applied normal to the sheet, whereas induced magnetic field is neglected by assuming low magnetic Reynolds number (see Fig. 1). Further, we have considered the constant temperature $T_{w}$ near the sheet and ambient temperature $T_{\infty}$.

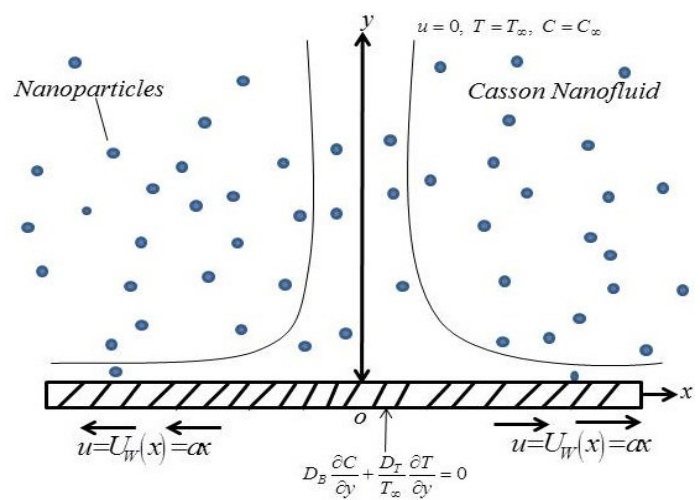

Fig. 1. Physical configuration and coordinate system

The boundary layer flow of a Casson nanofluid are govern as follows

$$
\begin{gathered}
u \frac{\partial u}{\partial x}+v \frac{\partial u}{\partial y}=v\left(1+\frac{1}{\beta}\right) \frac{\partial^{2} u}{\partial y^{2}}-\frac{\sigma B_{o}^{2}}{\rho} u-\frac{v}{k_{1}} u \\
u \frac{\partial T}{\partial x}+v \frac{\partial T}{\partial y}=\alpha_{m} \frac{\partial^{2} T}{\partial y^{2}}+\tau\left[D_{B} \frac{\partial C}{\partial y} \frac{\partial T}{\partial y}+\frac{D_{T}}{T_{\infty}}\left(\frac{\partial T}{\partial y}\right)^{2}\right]-\frac{1}{\left(\rho C_{p}\right)_{f}} \frac{\partial q_{r}}{\partial y} \\
u \frac{\partial C}{\partial x}+v \frac{\partial C}{\partial y}=D_{B} \frac{\partial^{2} C}{\partial y^{2}}+\frac{D_{T}}{T_{\infty}} \frac{\partial^{2} T}{\partial y^{2}}
\end{gathered}
$$

subject to boundary conditions as

$$
\begin{gathered}
u=U_{W}(x)=a x, v=0, T=T_{w}, D_{B} \frac{\partial C}{\partial y}+\frac{D_{T}}{T_{\infty}} \frac{\partial T}{\partial y}=0 \text { at } y=0 \\
u \rightarrow 0, v \rightarrow 0, T \rightarrow T_{\infty}, C \rightarrow C_{\infty} \text { as } y \rightarrow \infty
\end{gathered}
$$

According to Rosseland's approximation (Hussanan et al. [19]), the radiative heat flux is simplified as

$$
q_{r}=-\frac{4 \sigma^{*}}{3 k^{*}} \frac{\partial T^{4}}{\partial y}
$$

where $\sigma^{*}$ and $k^{*}$ are the Stefan-Boltzmann and mean absorption coefficient, respectively. By expanding $T^{4}$ in the Taylor series about $T_{\infty}$ and ignoring the higher order terms, we get

$$
T^{4} \square \quad-3 T_{\infty}^{4}
$$

By substituting equations (5) and (6) into equation (2), we get

$$
\begin{aligned}
u \frac{\partial T}{\partial x}+v \frac{\partial T}{\partial y} & =\alpha_{m} \frac{\partial^{2} T}{\partial y^{2}}+\tau\left[D_{B} \frac{\partial C}{\partial y} \frac{\partial T}{\partial y}+\frac{D_{T}}{T_{\infty}}\left(\frac{\partial T}{\partial y}\right)^{2}\right] \\
& +\frac{16 \sigma^{*} T_{\infty}^{3}}{3 k^{*}\left(\rho C_{p}\right)_{f}} \frac{\partial^{2} T}{\partial y^{2}}
\end{aligned}
$$

Follow Yasin et al. [20], we introduced the similarity transformations

$$
\begin{aligned}
& \psi=(a v)^{1 / 2} x f(\eta), \eta=\left(\frac{a}{v}\right)^{\frac{1}{2}} y, \\
& \theta(\eta)=\frac{T-T_{\infty}}{T_{w}-T_{\infty}}, \phi(\eta)=\frac{C-C_{\infty}}{C_{W}-C_{\infty}} .
\end{aligned}
$$

The stream function $\psi$ is defined as such that $u=\frac{\partial \psi}{\partial y}$ and $v=-\frac{\partial \psi}{\partial x}$. After the substitution of above transformations and equations (1), (3) and (7) take the following forms

$$
\begin{aligned}
& \left(1+\frac{1}{\beta}\right) f^{\prime \prime \prime}(\eta)+f^{\prime}(\eta) f^{\prime \prime}(\eta)-f^{\prime 2}(\eta) \\
& -\left(Q+k_{p}\right) f^{\prime}(\eta)=0 \\
& \frac{1}{\operatorname{Pr}}\left(1+\frac{4}{3} R\right) \theta^{\prime \prime}(\eta)+N b \theta^{\prime}(\eta) \phi^{\prime}(\eta)+N t \theta^{\prime 2} \\
& +f(\eta) \theta^{\prime}(\eta)=0 \\
& \phi^{\prime \prime}(\eta)+S c . f(\eta) \phi^{\prime}(\eta)+\frac{N t}{N b} \theta^{\prime \prime}(\eta)=0
\end{aligned}
$$

Where $f, \theta$ and $\phi$ are functions of $\eta$ and prime denotes derivatives with respect to $\eta$. The corresponding boundary conditions will follow the following form:

$$
\begin{gathered}
f(0)=0, f^{\prime}(0)=1, \theta(0)=1, \phi(0)=1, \\
f^{\prime}(\infty)=0, \theta(\infty)=0, \phi(\infty)=0 .
\end{gathered}
$$

The interests of physical quantities are skin friction coefficient $C_{f}$, local Nusselt number $N u_{x}$ and Sherwood number $S h_{x}$ which are defined as

$$
C_{f}=\frac{\tau_{w}}{\rho U_{W}^{2}}, N u_{x}=\frac{x q_{w}}{k T_{\infty}} \text { and } S h_{x}=\frac{x q_{m}}{D_{B}\left(C_{w}-C_{\infty}\right)} .
$$

where the shear stress $\tau_{w}$, surface heat flux $q_{w}$ and surface mass flux $q_{m}$ are given by

$$
\tau_{w}=\mu\left(\frac{\partial u}{\partial y}\right), q_{w}=-k \frac{\partial T}{\partial y} \text { and } q_{m}=-D_{B} \frac{\partial C}{\partial y} \text { at } y=0 .
$$

Using the similarity transformations given in equation (8), we obtain 


$$
C_{f}\left(\operatorname{Re}_{x}\right)^{\frac{1}{2}}=f^{\prime \prime}(0), \frac{N u_{x}}{\left(\operatorname{Re}_{x}\right)^{\frac{1}{2}}}=-\theta^{\prime}(0) \text { and } \frac{S h_{x}}{\left(\operatorname{Re}_{x}\right)^{\frac{1}{2}}}=-\phi^{\prime}(0) \text {. }
$$

where $\operatorname{Re}_{x}=\frac{x U_{w}(x)}{v}$ defined as local Reynold's number.

\section{RESULT AND DISCUSSIONS}

The graphical results for temperature $\theta(\eta)$, velocity $f^{\prime}(\eta)$ and concentration $\phi(\eta)$ profiles for different involved parameters such as Casson parameter $\beta$, magnetic parameter $M$, porosity parameter $k_{p}$, radiation parameter $R$, Prandlt number Pr, Brownian motion parameter $N b$ and thermophoresis parameter $N t$. The non-linear ordinary differential equations are derived manually together with boundary conditions. Then, these equations are solved by using Shooting method. Those non-linear ordinary differential equations are of second order in $\theta$ and $\phi$, third order in $f$, are then reduced into simultaneous ordinary equations. With the initial conditions of $\eta \rightarrow \infty$, we can get the value of $f^{\prime}(\eta), \theta(\eta)$ and $\phi(\eta)$. Thus, we may obtain unknown initial conditions $\eta=0$ by using Shooting method and assumed the initial values for boundary value problem.

Table 1. Comparison of results for the local Nusselt number $-\theta^{\prime}(0)$ when

\begin{tabular}{ccccc}
\multicolumn{5}{c}{$\beta=\infty, L e=1, R=K=M=N b=N t=0}$. \\
\hline Pr & $\begin{array}{c}\text { Khan and } \\
\text { Pop [25] }\end{array}$ & $\begin{array}{c}\text { Gorla and } \\
\text { Sidawi } \\
{[26]}\end{array}$ & $\begin{array}{c}\text { Wang } \\
{[27]}\end{array}$ & $\begin{array}{c}\text { Present } \\
\text { study }\end{array}$ \\
\hline 0.20 & 0.1691 & 0.1691 & 0.1691 & 0.1698 \\
0.70 & 0.4539 & 0.5349 & 0.4539 & 0.4539 \\
2.00 & 0.9113 & 0.9114 & 0.9114 & 0.9113 \\
7.00 & 1.8954 & 1.8905 & 1.8954 & 1.8954 \\
20.00 & 3.3539 & 3.3539 & 3.3539 & 3.3539 \\
\hline
\end{tabular}

The calculated boundary values should be similar with the real boundary values, hence, the value should as close to the boundary values are appropriate to consider. In this study, there are no consideration of variation in temperature, velocity and concentration where we took large infinity condition but finite value for $\eta$. We chose the value of $\eta_{\max }=8$, which is appropriate and sufficient to achieve asymptotic for all examined parameter with the boundary conditions. Table 1 show the comparison values of $-\theta^{\prime}(0)$ with previous results. It concluded that this method work efficiently and the results presented here are accurate.

The effects of Casson parameter $\beta$ on velocity and temperature profiles are shown in Fig. 2 and 3. It is found that an increase in Casson parameter $\beta$ leads to velocity profiles decrease but temperature profiles increase. When increase the values of Casson parameter $\beta$ means to reduce yield stress, thus Casson fluid behaves like Newtonian fluid $(\beta \rightarrow \infty)$. It is also concluded that Casson fluid velocity is greater than that of Newtonian fluid.

Fig. 4 and 5 show that the magnetic parameter $M$ effects on velocity and temperature profiles, respectively. The velocity profiles drops when magnetic parameter increases. This is because when magnetic parameter increases, the Lorentz force also will increase and caused to oppose the fluid motion at the same time. Fig. 5 shows that an increase in the magnetic parameter $M$ leads to an increase in temperature. Further, this enhancement is very significant near the sheet, however this effect is almost negligible away from the sheet.

Fig. 6 and 7 illustrated the temperature and concentration profiles for different values of Brownian parameter $N b$. It is observed that increasing value of $\mathrm{Nb}$ cause the temperature field increases but the concentration profiles decreases. Since Brownian motion refers to the particle movement, hence, the active the movement of particles, the more heat produced and temperature will rise. Fig. 8 and 9 demonstrated the temperature and concentration profiles of thermophoresis parameter respectively. It is observed that increasing the value of $N t$ resulted the temperature and concentration profiles increase.

Fig. 10 reveals the temperature profile of radiation parameter. Based on the graph, the result shown that increase the value of $R$ then wall temperature increase as well. Fig. 11 and 12 is plotted to explain the effect of Casson nanofluid and Magnetic parameter on local Nusselt number and skin friction coefficient, respectively. The greater the value of Casson parameter will cause the higher temperature gradient. But, with the larger value of magnetic force and it will reduce the thickness of boundary layer. Fig. 12 shows that the skin friction coefficient decreases when the value of Casson parameter and magnetic parameter increase. Influence of Brownian motion parameter and thermophoresis parameter on local Nusselt number is illustrated in Fig. 13. It can be seen that the dimensionless temperature rate decreases at all time when increases the value of $\mathrm{Nb}$ and Nt. Figure 14 shows the effect of Prandlt number and radiation parameter on temperature gradient. With increasing the Pr value, it is also increase the local Nusselt number. However, the change of heat transfer decreases once the radiation parameter increases. 


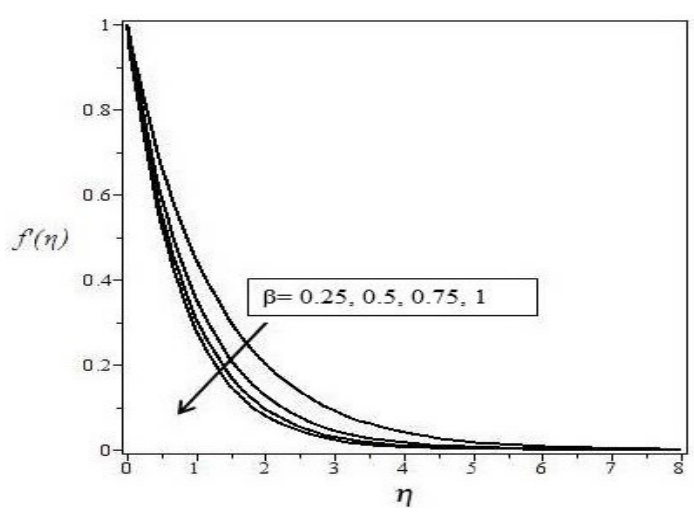

Fig. 2. Velocity profiles for various values of Casson parameter $\beta$, when $M=0.2, K=2, R=5, N b=0.5$, $N t=0.5, S c=2$ and $\operatorname{Pr}=7$.

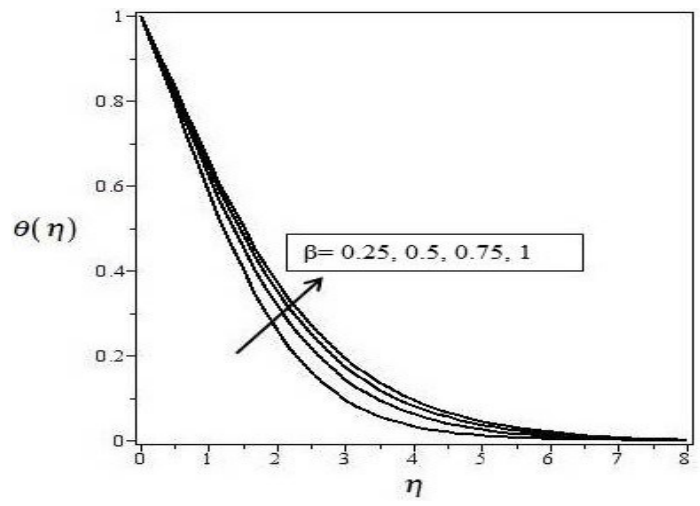

Fig. 3. Temperature profiles for various values of Casson parameter $\quad \beta$, when $\quad M=0.2, K=2, R=5$, $N b=0.5, N t=0.5, S c=2$ and $\operatorname{Pr}=7$.

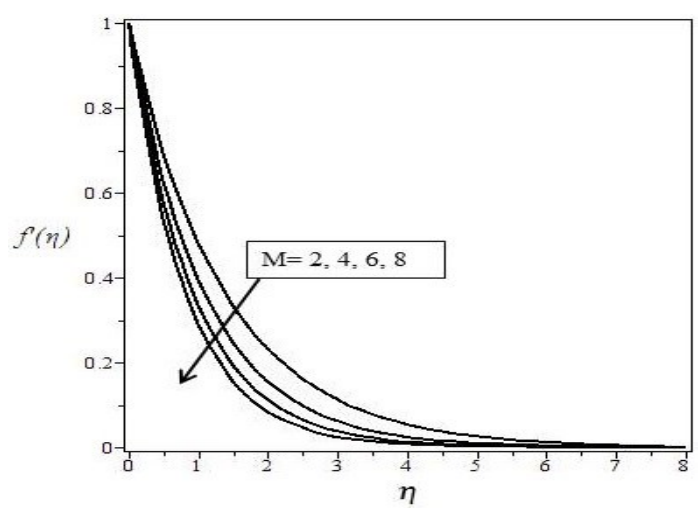

Fig. 4. Velocity profiles for various values of magnetic parameter $M$, when $\beta=0.2, K=2, R=5$, $N b=0.5, N t=0.5, S c=2$ and $\operatorname{Pr}=7$.

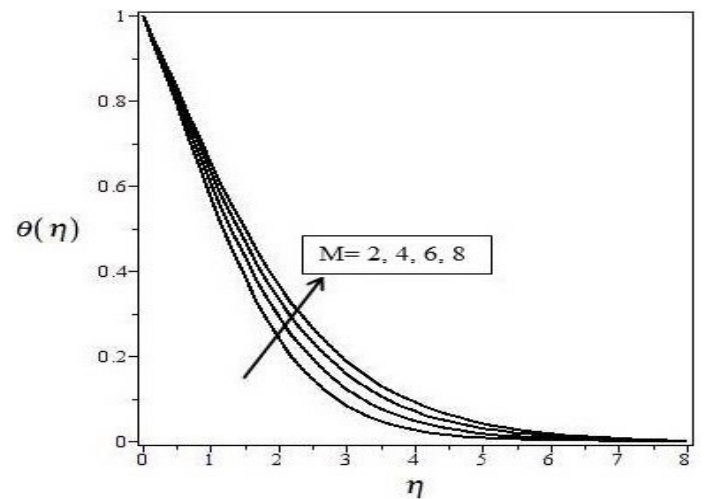

Fig. 5. Temperature profiles for various values of magnetic parameter $M$, when $\beta=0.2, K=2, R=5$, $N b=0.5, N t=0.5, S c=2$ and $\operatorname{Pr}=7$.

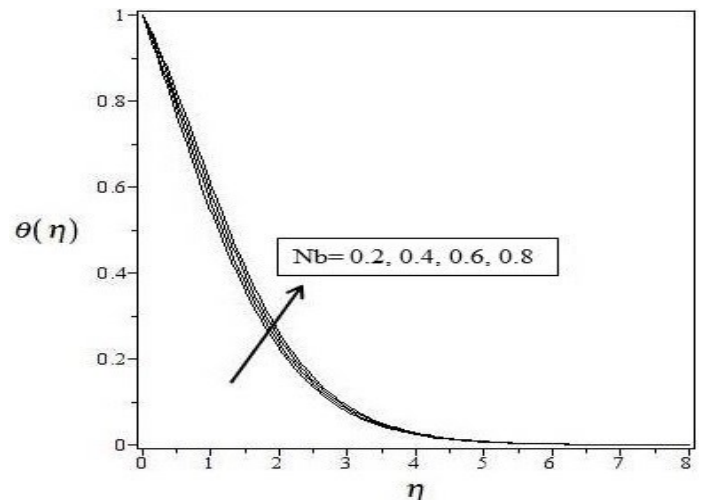

Fig. 6. Temperature profiles for various values of Brownian motion parameter $N b$, when $M=0.2, K=2$, $R=5, \beta=0.2, N t=0.5, S c=2$ and $\operatorname{Pr}=7$.

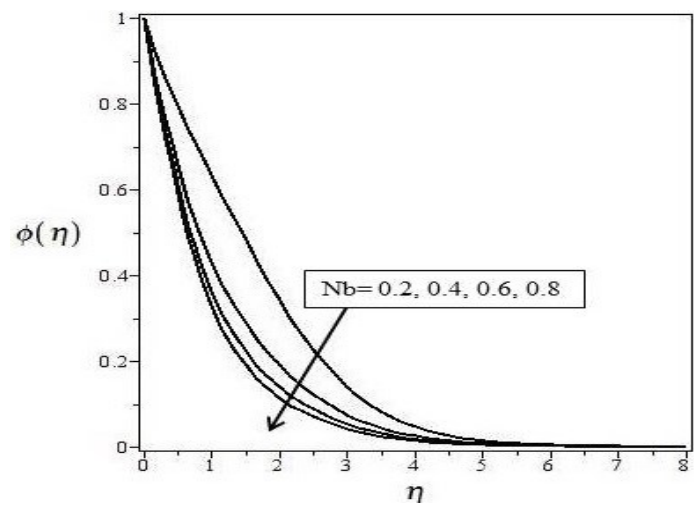

Fig. 7. Concentration profiles for various values of Brownian motion parameter $N b$, when $M=0.2, K=2$, $R=5, \beta=0.2, N t=0.5, S c=2$ and $\operatorname{Pr}=7$. 


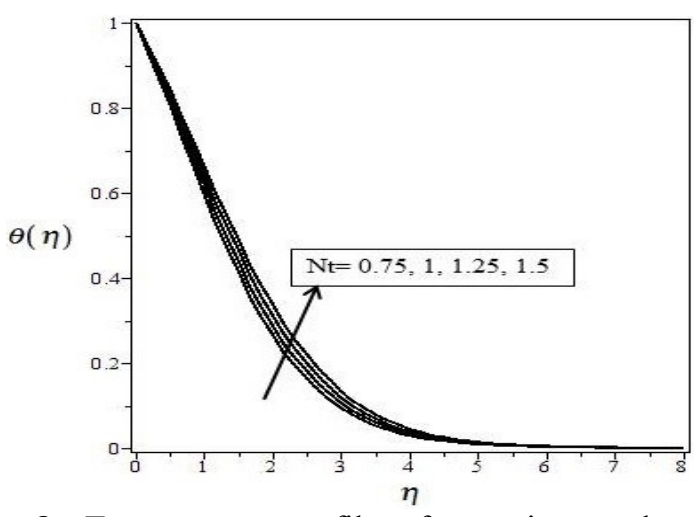

Fig. 8. Temperature profiles for various values of thermophoresis parameter $N t$, when $M=0.2, K=2$, $R=5, \beta=0.2, N b=0.5, S c=2$ and $\operatorname{Pr}=7$.

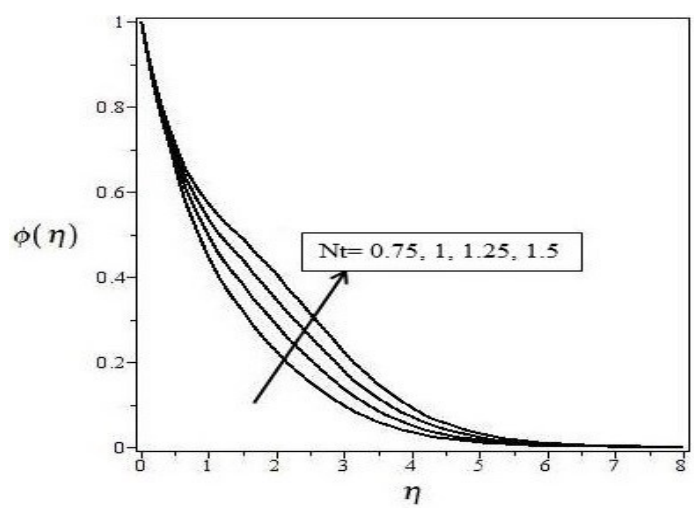

Fig. 9. Concentration profiles for various values of thermophoresis parameter $N t$, when $M=0.2, K=2$, $R=5, \beta=0.2, N b=0.5, S c=2$ and $\operatorname{Pr}=7$.

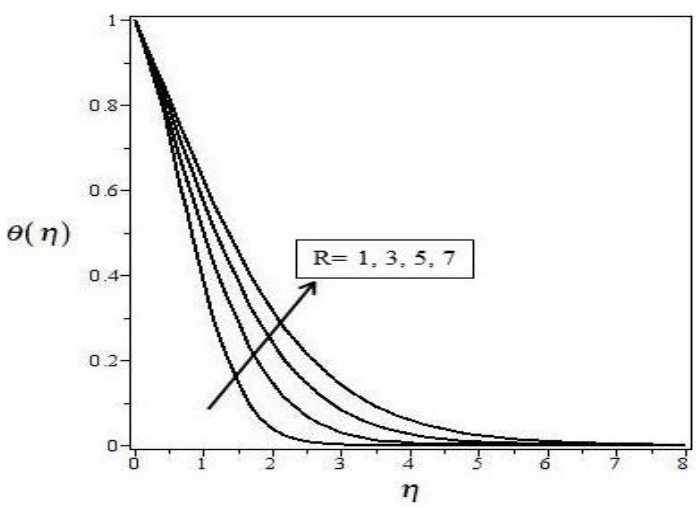

Fig. 10. Temperature profiles for various values of radiation parameter $\operatorname{Pr}$, when $M=0.2, K=2$, $\operatorname{Pr}=7, N t=0.5, \beta=0.2, N b=0.5$ and $S c=2$.

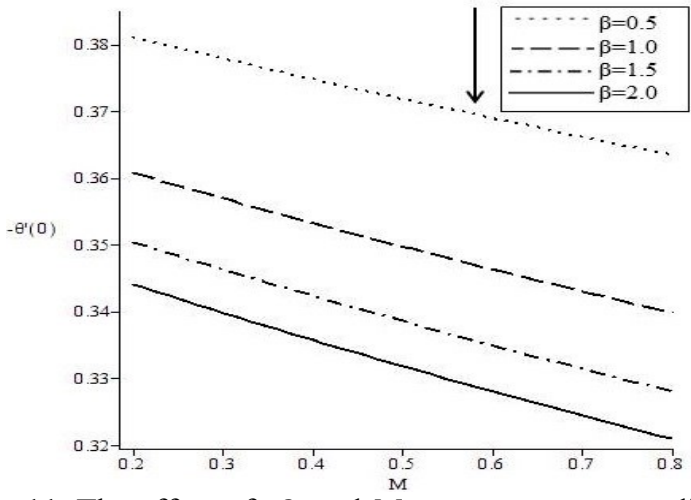

Fig. 11. The effect of $\beta$ and $M$ on temperature gradient.

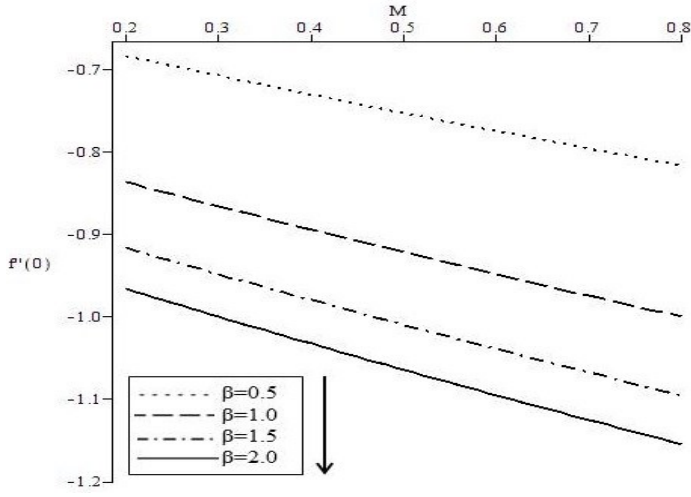

Fig. 12. The effect of $\beta$ and $M$ on skin friction coefficient.

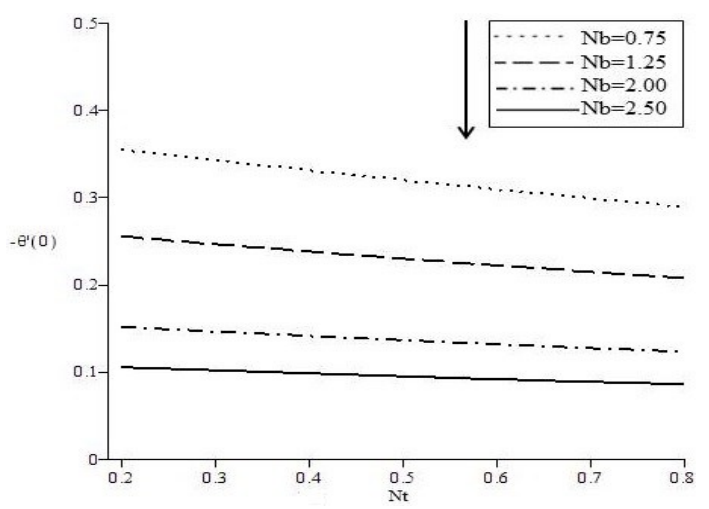

Fig 13. The effect of $N b$ and $N t$ on temperature gradient.

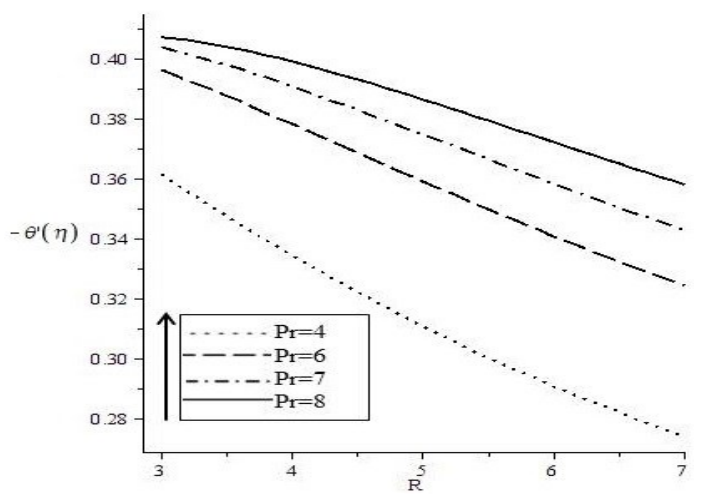

Fig. 14. The effect of $\operatorname{Pr}$ and $R$ on temperature gradient. 


\section{Conclusions}

Thermal radiation effects on MHD flow and heat mass transfer over stretching sheet in Casson nanofluid is studied numerically. The nonlinear ordinary differential equations are solved numerically using the Shooting method. The result of velocity, temperature and concentration profiles are then presented graphically for different values of the pertinent parameters. Also, the skin friction coefficient, local Nusselt number and Sherwood number are examined with various parameters. The results are summarized as below:

- Boundary layer thickness increase in temperature and concentration profiles but velocity profiles decreases when Casson nanofluid parameter, magnetic parameter and porosity parameter increase.

- Increase in Brownian motion parameter causes the concentration profiles to drop but the wall temperature increases.

- Increases thermophoresis parameter also causes temperature and concentration profiles increase.

- Temperature profile increases as the radiation parameter increases.

- Skin friction coefficient and local Nusselt number decreases at all the time as $\beta$ and $M$ increase in the fluid.

- Local Nusselt number decreases and Sherwood number increases as $N b$ and $N t$ increase in the fluid.

- The higher the value of $\operatorname{Pr}$ and $R$, the lower the value of local Nusselt number.

We would like to thank Universiti Malaysia Pahang for the financial support from Ministry of Higher Education Malaysia (RDU170358 and RDU150101).

\section{References}

1. S. Choi, ASME. 66, 7 (1995)

2. J. Buongiorno, J. Heat Trans-t ASME 128, 11 (2006)

3. S. Ahmed, I. Pop, Int. Commun. Heat Mass 37, 5 (2010)

4. R.K. Tiwari, M.K. Das, Int. J. Heat Mass Trans. 50, 9 (2007)

5. N.A. Yacob, A. Ishak, I. Pop, K. Vajravelu, Nanoscale Research Letters. 6, 8 (2011)

6. A.J. Chamkha, A.M. Aly, Chem. Eng. Commun. 198, 17 (2011)

7. A. Noghrehabadi, R. Pourrajab, M. Ghalambaz, Int. J. Therm. Sci. 54, 28 (2012)

8. A. Mahdy, Nucl. Eng. Des. 249, 8 (2012)

9. M. Qasim, I. Khan, S. Shafie, Math. Probl. Eng. 7 (2013)
10. P. Loganathan, C. Vimala, Indian J. Phy. 88, 5 (2014)

11. B. Ganga, S.M.Y. Ansari, N.V Ganesh, A.K.A. Hakeem, J. Taibah Uni. Sci. (2016)

12. M.A.A. Hamad, I. Pop, A.I.M. Ismail, Nonlinear Analysis: Real World Applications. 12, 19 (2011)

13. R. Ellahi, Appl. Math. Model. 37, 17 (2013)

14. M. Qasim, Z.H. Khan, W.A. Khan, I.A. Shah, Plos One. 9, (2014)

15. A. Hussanan, I. Khan, H. Hashim, M.K.A Anuar, N. Ishak, N.M. Sarif, M.Z. Salleh, J. Teknologi. 78, 6 (2016)

16. P.M. Krishna, V. Sugunamma, N. Sandeep, Chem. Process Eng. Res. 25, 14 (2014)

17. R.U. Haq, Z.H. Khan, W.A. Khan, Phys. Eng. 63, 8 (2014)

18. M.Y. Malik, M. Naseer, S. Nadeem, A. Rehman, Appl. Nanoscience. 4, 5 (2014)

19. A. Hussanan, M.Z. Salleh, I. Khan, R.M. Tahar, Z. Ismail, Maejo Int. J. Sci. Tech. 9, 22 (2015)

20. M.H.M. Yasin, A. Ishak, I. Pop, J. of Magnetism and Magnetic Materials. 407, 6 (2016)

21. W.A. Khan, I. Pop, Int. J. Heat Mass Trans. 53, 7 (2010)

22. R.S.R. Gorla, I. Sidawi, Appl. Scientific Res. 52, 11 (1994)

23. C.Y. Wang, ZAMM J. of Appl. Math. and Mechanics, 69, 3 (1989) 